\title{
Phase Noise Cancellation in Coherent Communication Systems Using a Radio Frequency Pilot Tone
}

\author{
Tianhua Xu ${ }^{1,2,3, * \mathbb{C}}$, Cenqin Jin ${ }^{2}$, Shuqing Zhang ${ }^{4, *}$, Gunnar Jacobsen ${ }^{5,6}$, Sergei Popov ${ }^{6}$, \\ Mark Leeson ${ }^{2}\left(\mathbb{D}\right.$ and Tiegen Liu ${ }^{1}$ \\ 1 Key Laboratory of Opto-Electronic Information Technology (MoE), School of Precision Instruments and \\ Opto-Electronics Engineering, Tianjin University, Tianjin 300072, China; tgliu@tju.edu.cn \\ 2 School of Engineering, University of Warwick, Coventry CV4 7AL, UK; Cenqin.Jin@warwick.ac.uk (C.J.); \\ Mark.Leeson@warwick.ac.uk (M.L.) \\ 3 Optical Networks Group, University College London, London WC1E 7JE, UK \\ 4 Department of Optical Engineering, Harbin Institute of Technology, Harbin 150001, China \\ 5 NETLAB, RISE Research Institutes of Sweden, SE-16440 Stockholm, Sweden; gunnar.jacobsen@ri.se \\ 6 Optics and Photonics Group, KTH Royal Institute of Technology, SE-16440 Stockholm, Sweden; \\ sergeip@kth.se \\ * Correspondence: tianhua.xu@ieee.org (T.X.); sq.zhang@hit.edu.cn (S.Z.)
}

Received: 26 September 2019; Accepted: 3 November 2019; Published: 5 November 2019

Featured Application: This work is performed for the compensation of the laser phase noise (LPN) and the equalization enhanced phase noise (EEPN) in high-capacity, long-haul, coherent optical fiber networks.

\begin{abstract}
Long-haul optical fiber communication employing digital signal processing (DSP)-based dispersion compensation can be distorted by the phenomenon of equalization-enhanced phase noise (EEPN), due to the reciprocities between the dispersion compensation unit and the local oscillator (LO) laser phase noise (LPN). The impact of EEPN scales increases with the increase of the fiber dispersion, laser linewidths, symbol rates, signal bandwidths, and the order of modulation formats. In this work, the phase noise cancellation (PNC) employing a radio frequency (RF) pilot tone in coherent optical transmission systems has been investigated. A 28-Gsym/s QPSK optical transmission system with a significant EEPN has been implemented, where the carrier phase recovery (CPR) was realized using the one-tap normalized least-mean-square (NLMS) estimation and the differential phase detection (DPD), respectively. It is shown that the RF pilot tone can entirely eliminate the LPN and efficiently suppress the EEPN when it is applied prior to the CPR.
\end{abstract}

Keywords: coherent optical fiber communication; laser phase noise (LPN); carrier phase recovery (CPR); phase noise cancellation (PNC); equalization enhanced phase noise (EEPN); radio frequency (RF) pilot tone

\section{Introduction}

Long-haul optical communication is seriously deteriorated by transmission impairments, e.g., chromatic dispersion (CD), polarization mode dispersion (PMD), laser phase noise (LPN), and Kerr fiber nonlinearities [1,2]. The combination of coherent detection, digital signal processing (DSP), and advanced modulation formats offers a very promising solution for long-haul, high-capacity optical transmission, to offer great capabilities and flexibilities in the design, deployment, and operation of core telecommunication networks [3-5]. In the reported phase noise cancellation (PNC) methods, the radio frequency (RF) pilot tone scheme was verified to be an efficient approach to remove laser phase 
fluctuations [6-9]. However, these works only studied the behaviors of RF pilot tones in short-reach systems, where the enhancement effect of fiber dispersion on the LPN was neglected [10,11]. Actually, due to the interplay between the electronic dispersion compensation (EDC) module and the LPN from the local oscillator (LO), a phenomenon of equalization enhanced phase noise (EEPN) is induced and plays a significant role in the carrier phase recovery (CPR) in high-capacity optical communication systems [10-16]. However, so far, no DSP-based CPR has been developed to effectively compensate for EEPN [16-18]. Therefore, it will be of great significance to study the suppression of LPN and EEPN using an RF pilot tone scheme.

In this work, the performance of an orthogonally polarized RF pilot tone scheme is investigated for eliminating both the LPN and the EEPN in long-haul optical transmission systems. A 28-Gsym/s quadrature phase shift keying (QPSK) system is numerically implemented, where the CPR is realized using a one-tap normalized least mean square (NLMS) estimation and a differential phase detection (DPD) scheme, respectively [19-21]. The results show that the LPN can be fully removed and the EEPN can also be effectively suppressed, when the RF pilot tone scheme is applied prior to the CPR.

\section{EEPN in Optical Communication Systems}

Figure 1 describes the block diagram of a long-haul, coherent optical communication system with EDC and CPR. The LPN from the transmitter (Tx) laser passes through the optical fiber and the dispersion compensation unit, and thus the net CD experienced by the Tx LPN approaches zero. By contrast, the LO LPN goes only through the dispersion compensation unit. Consequently, the LO LPN interplays with the dispersion compensation component in EDC and the interactions will degrade the performance of the optical communication system. This effect is called EEPN [10-13].

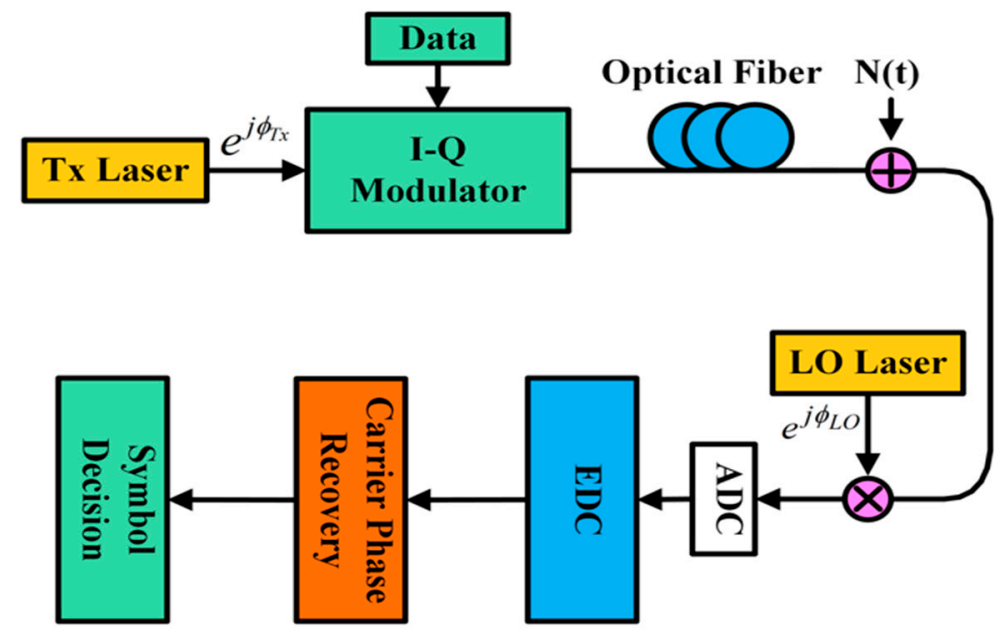

Figure 1. Block diagram of coherent transmission system and equalization-enhanced phase noise $(\mathrm{EEPN}) . \phi_{T x}$ : Tx laser phase noise (LPN), $\phi_{L O}$ : LO LPN, ADCs: analogue-to-digital converters.

The variance of the EEPN distortion will increase with fiber dispersion, local oscillator laser linewidth, and signal symbol rate. The noise variance of EEPN can be written as follows $[10,19]$ :

$$
\sigma_{E E P N}^{2}=\frac{\pi \lambda^{2}}{2 c} \cdot \frac{D \cdot L \cdot \Delta f_{L O}}{T_{S}}
$$

where $\lambda$ is the central wavelength of the optical carrier, $c$ is the speed of the light in vacuum, $L$ is the length, $D$ is the CD coefficient of the fiber, $T_{S}$ is the symbol period of the signal, and $\Delta f_{L O}$ is the 3-dB linewidth of the LO laser.

It is noted that the EEPN evaluation in Equation (1) only works for the static time-domain and frequency-domain EDCs, which involve no phase noise compensation functions [22,23]. 


\section{CPR Using One-Tap NLMS}

A one-tap NLMS filter could be effectively applied in the CPR [20], and its tap weight $w(k)$ is expressed using the following equations:

$$
\begin{gathered}
w(k+1)=w(k)+\frac{\mu}{|x(k)|^{2}} x^{*}(k) e(k), \\
e(k)=d(k)-w(k) \cdot x(k),
\end{gathered}
$$

where $x(k)$ is the input signal, $k$ is the symbol index, $d(k)$ is the desired symbol, $e(k)$ is the error between the output and the desired symbols, and $\mu$ represents a parameter of the step size.

The schematic of the NLMS CPR is illustrated in Figure 2, which can actually be implemented in a feed-forward structure and can be realized in parallel in the field programmable gate array (FPGA) circuits for a real-time operation [4].

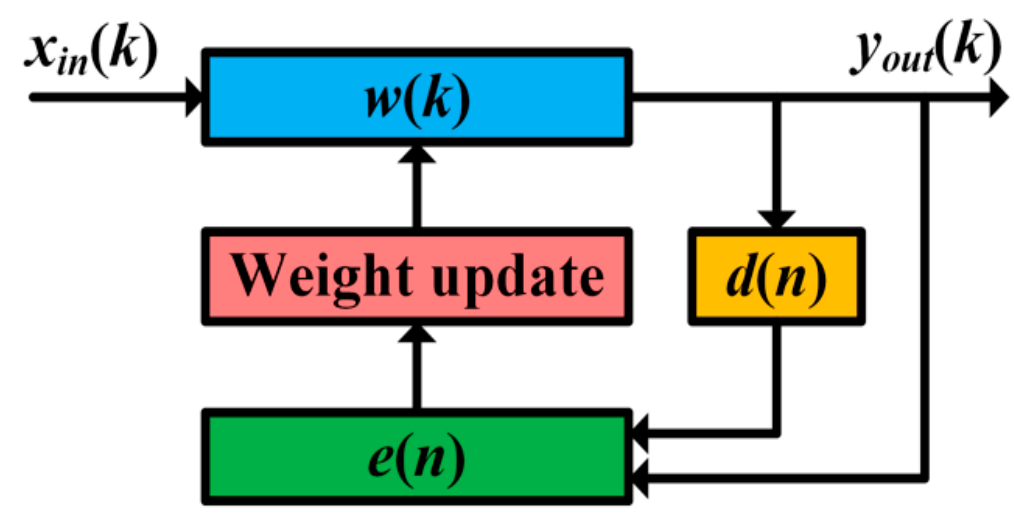

Figure 2. Block diagram of the one-tap normalized least-mean-square (NLMS) carrier phase recovery (CPR).

Similar to the definition of the Tx and the LO, a concept of effective linewidth $\Delta f_{E f f}$ is employed here to describe the total phase noise in coherent transmission systems considering EEPN $[17,19]$ :

$$
\begin{gathered}
\Delta f_{E f f} \approx \frac{\sigma_{T x}^{2}+\sigma_{L O}^{2}+\sigma_{E E P N}^{2}}{2 \pi T_{S}} \\
\sigma_{T x}^{2}=2 \pi \Delta f_{T x} \cdot T_{S} \\
\sigma_{L O}^{2}=2 \pi \Delta f_{L O} \cdot T_{S}
\end{gathered}
$$

where $\sigma_{T x}^{2}$ and $\sigma_{L O}^{2}$ are the LPN variance of the Tx and LO lasers, respectively, and $\Delta f_{T x}$ is the 3-dB linewidth of the Tx laser.

It has been established that the step size $\mu$ can be optimized to enhance the performance of NLMS CPR [19]. Therefore, it is significant to find the optimal step size for NLMS CPR, when the EEPN is considered in coherent optical communication systems. Figure 3 shows the optimal step size parameter for various effective linewidths in the 28-Gsym/s QPSK system, applicable to the time-domain and the frequency-domain static dispersion equalizations [22,23]. In all numerical simulations in this paper, the NLMS algorithm is operated with its corresponding optimal value of the step size. 


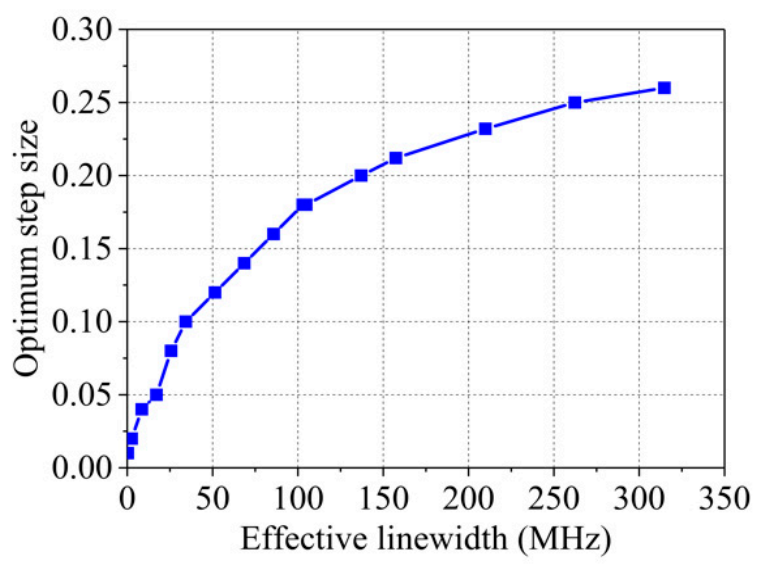

Figure 3. The optimal step size parameter in the NLMS CPR for various effective linewidths.

\section{Differential Carrier Phase Recovery}

Differential phase detection (DPD) can also be employed for recovering the carrier phase in coherent communication systems. In the DPD scheme, signal data are encoded in and extracted from the phase differences between consecutive transmitted signal symbols [21]. For instance, the phase information of the $k$-th symbol can be extracted according to the phase difference between the $k$-th and the $(k+1)$-th symbols. Therefore, the CPR error is determined by the phase fluctuation between the $k$-th and the $(k+1)$-th symbols within a symbol period [19]. It is noted that DPD does not require additional computations such as the $n$-power, the averaging, and the phase unwrapping, compared to other CPR methods [20,24-26]. Therefore, it can be easily implemented in DSP hardware for a real-time operation. The block diagram of DPD CPR is provided in Figure 4.

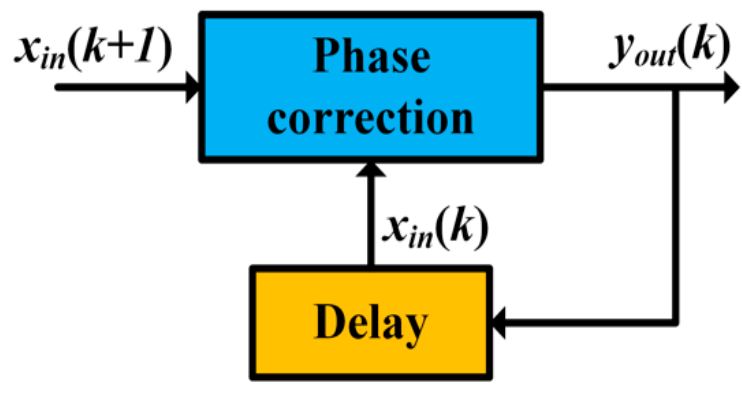

Figure 4. Schematic of the differential phase detection.

\section{Transmission Setup with RF Pilot Tone Scheme}

Figure 5 depicts a 28-Gsym/s QPSK coherent optical fiber transmission system using an RF pilot tone orthogonally polarized against the transmitted signals. The electrical data were converted into 28-Gsym/s QPSK signals using an in-phase and quadrature (I-Q) optical modulator. The modulated signals occupied one polarization state, and the RF pilot tone was transmitted in the orthogonal polarization state. The signals and the RF pilot tone are integrated into the fiber via a polarization beam combiner (PBC). At the receiver side, both the received signals and the RF pilot tone are mixed with the LO laser, respectively, and are then detected by balanced photodiodes after the $90^{\circ}$ hybrid. After that, both the received signals and the RF pilot tone are digitized using 8-bit analogue-to-digital converters (ADCs) at $56 \mathrm{Gsym} / \mathrm{s}$ and are then equalized using the EDC module. The signals are further multiplied with a conjugate of the processed RF pilot tone to suppress the impact of both LPN and EEPN. The central wavelengths of both the transmitter and the local oscillator lasers are $1553.6 \mathrm{~nm}$, and the fiber dispersion coefficient is $16 \mathrm{ps} \cdot \mathrm{nm}^{-1} \mathrm{~km}^{-1}$. Here, we have neglected fiber attenuation, 
PMD, and Kerr nonlinearities $[27,28]$. The EDC is implemented in the frequency domain [23]. The CPR is performed using the one-tap NLMS and DPD methods, respectively.

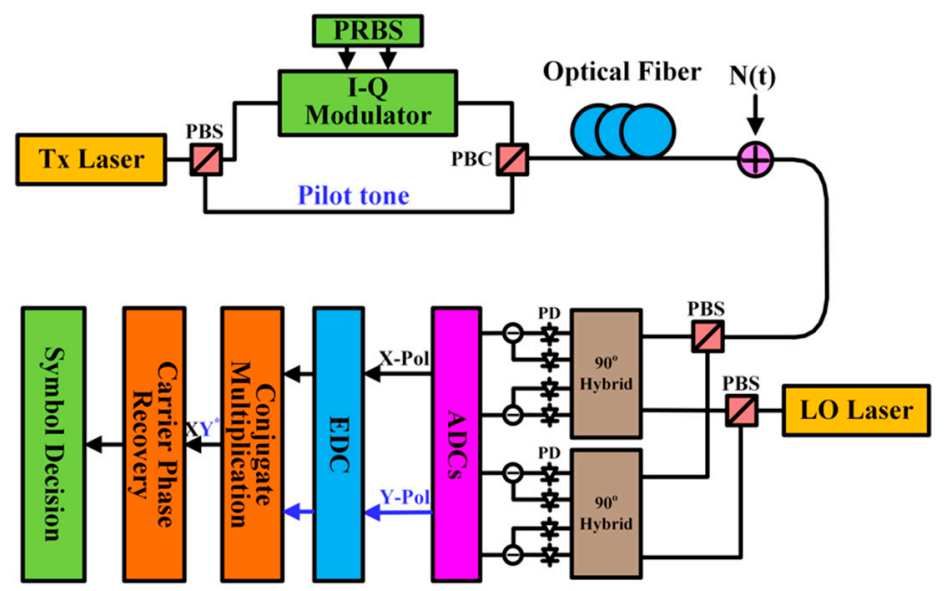

Figure 5. High-speed coherent communication system with an orthogonally-polarized RF pilot tone.

\section{Results and Analyses}

Figure 6 shows the results of PNC using the RF pilot tone in a $2000 \mathrm{~km}$ coherent communication system, where the CPR is realized using the NLMS algorithm. In Figure 6a, the effective linewidth is $170 \mathrm{MHz}$ and there is no EEPN, since the linewidth of the transmitter laser is $170 \mathrm{MHz}$ and the linewidth of the LO laser is $0 \mathrm{~Hz}$. It is found that both the Tx and the LO LPN can be entirely suppressed. In Figure 6b, the linewidths of both the Tx and the LO lasers are $5 \mathrm{MHz}$. According to Equations (1) and (4), the EEPN is quite significant in such a case, and the effective linewidth is the same $(170 \mathrm{MHz})$ as that in Figure $6 \mathrm{a}$. It is found in Figure $6 \mathrm{~b}$ that the BER performance improves considerably (around half an order of magnitude in the BER floor, from $8 \times 10^{-4}$ to $3 \times 10^{-4}$ ) using the RF pilot tone, compared to the case of NLMS CPR only. The results in Figure $6 \mathrm{a}, \mathrm{b}$ demonstrate the efficiency of the RF pilot tone in compensating for both LPN and EEPN. It can be found that the use of RF pilot tone can entirely mitigate the LPN from both the Tx and the LO lasers. However, it cannot fully compensate for the EEPN in optical fiber transmission systems since the EEPN actually represents a complicated integration of the phase, the amplitude, and the time jitter noise [10,12,16,29].

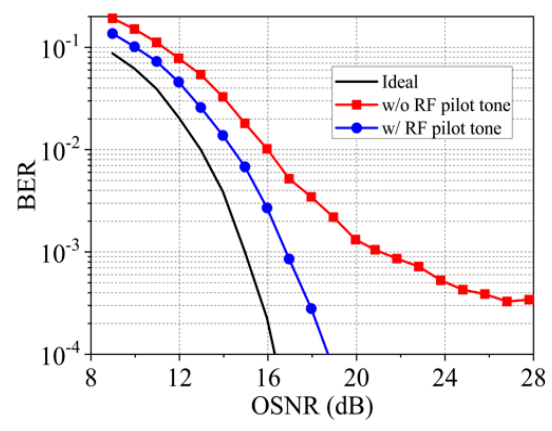

(a)

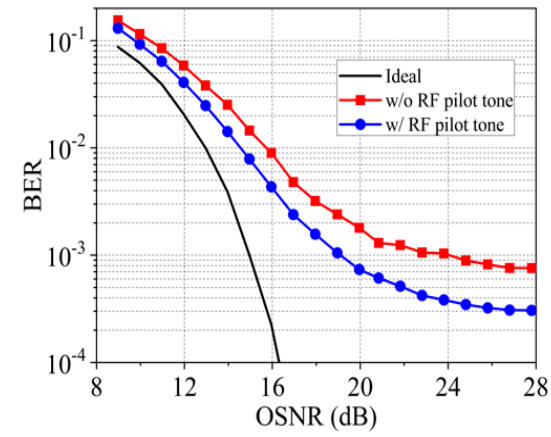

(b)

Figure 6. Phase noise cancellation (PNC) results of the $2000 \mathrm{~km}$ coherent transmission system (the one-tap NLMS CPR). Ideal: linewidth of both Tx and LO lasers is $0 \mathrm{~Hz}$. w/o: without, w/: with. (a) Transmitter: $170 \mathrm{MHz}$, local oscillator: $0 \mathrm{~Hz}$; (b) Transmitter = local oscillator: $5 \mathrm{MHz}$.

When the CPR is implemented using DPD, the results of the PNC using the RF pilot tone in the same $2000 \mathrm{~km}$ coherent system are illustrated in Figure 7. In Figure 7a, the linewidth of the transmitter laser is $170 \mathrm{MHz}$ and the linewidth of the LO laser is $0 \mathrm{~Hz}$, representing an absence of EEPN. It is found 
that the full suppression of LPN can be performed with the use of the RF pilot tone, compared to the scheme of DPD only. In Figure 7b, the linewidths of both the transmitter and local oscillator lasers are $5 \mathrm{MHz}$, so that the EEPN is significant again (with an effective linewidth of $170 \mathrm{MHz}$ ). Similar to Figure $6 \mathrm{~b}$, a considerable improvement of BER performance is also achieved with half an order of magnitude in the BER floor (from $6.5 \times 10^{-4}$ to $1.5 \times 10^{-4}$ ), when the RF pilot tone scheme is applied prior to the DPD.

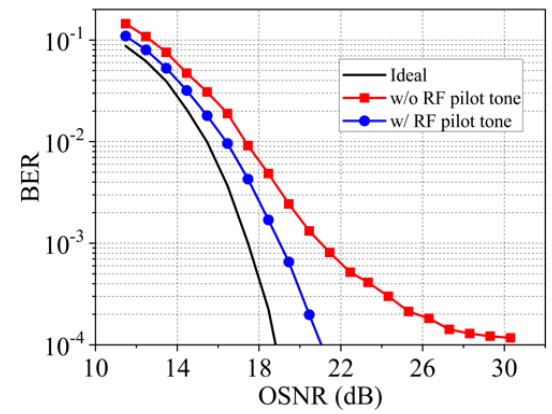

(a)

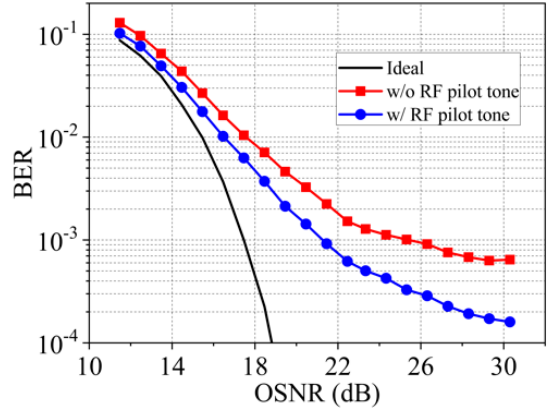

(b)

Figure 7. PNC results of the $2000 \mathrm{~km}$ coherent transmission system (DPD CPR). Ideal: linewidth of both Tx and LO lasers are $0 \mathrm{~Hz}$. (a) Transmitter: $170 \mathrm{MHz}$, local oscillator: $0 \mathrm{~Hz}$; (b) Transmitter = local oscillator: $5 \mathrm{MHz}$.

\section{Discussions}

It has to be clarified that the use of the RF pilot tone for the PNC in this work has occupied one polarization state in the fiber, since here we aim to investigate and discuss the efficiency of the use of an RF pilot tone for PNC in a simplified transceiver structure. In fact, the RF pilot tone can also be transmitted using the same polarization state as the optical data signals, which requires a more complicated implementation to perform the generation and recovery of the RF pilot tone $[7,30,31]$. In such systems, a pilot-carrier vector modulation (PCVM) scheme has been applied, where the transverse magnetic (TM) component is loaded with the signal data in X-polarization and the transverse electric (TE) component is employed to carry both the signal data in Y-polarization and the RF pilot tone [7]. It was reported that the PNC in such PCVM transmission schemes can also provide a good performance for an effective linewidth of up to $30 \mathrm{MHz}[7,31]$. The performance comparison between such PCVM transmission scheme and our proposed RF pilot tone-PNC scheme will be investigated in future work. In addition, for orthogonal frequency division multiplexing (OFDM) optical transmission systems it will be straightforward to employ one subcarrier as the RF pilot tone within the OFDM spectrum to remove the influence of laser phase noise and EEPN [32].

To study the impact of PMD on the proposed RF pilot tone PNC scheme, numerical simulations using the same 28-Gsym/s QPSK coherent transmission setup have been implemented with a PMD coefficient of $0.1 \mathrm{ps} / \sqrt{\mathrm{km}}$. The differential group delay (DGD) and the random rotations between the transmitted data and the RF pilot tone (due to effect of PMD) are mitigated using a constant modulus algorithm CMA equalizer $[5,33,34]$. The CPR is performed using the one-tap NLMS approach. Figure 8 shows the PNC results of the same $2000 \mathrm{~km}$ coherent system, with and without including the impact of PMD. Similarly, the linewidth of the Tx laser is $170 \mathrm{MHz}$ and the linewidth of the LO laser is $0 \mathrm{~Hz}$ in Figure 8a, and the linewidths of both the Tx and the $\mathrm{LO}$ lasers are $5 \mathrm{MHz}$ in Figure 8b. Both scenarios indicate an effective linewidth of $170 \mathrm{MHz}$, and there is no EEPN in Figure 8a. It can be clearly found that, for both transmission scenarios, the impact of PMD can be fully suppressed using the CMA equalizer and the performance of the RF pilot tone-based PNC (both with and without EEPN) will not be affected due to the introduction of PMD. 


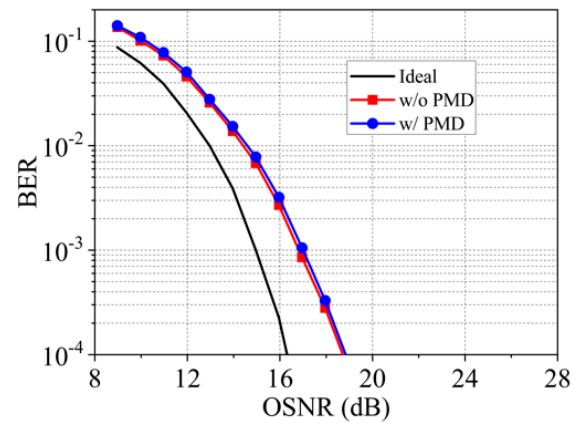

(a)

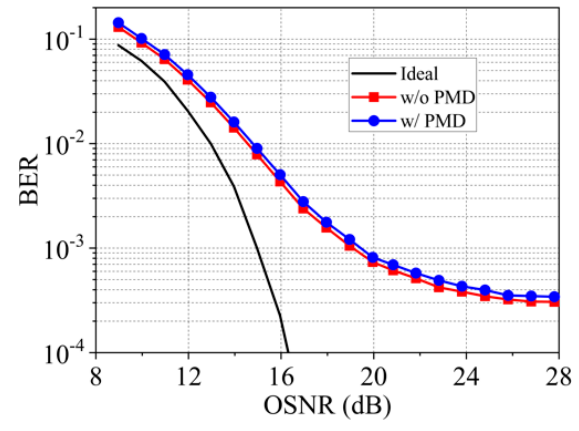

(b)

Figure 8. PNC performance of the $2000 \mathrm{~km}$ coherent transmission systems with and without PMD, when the NLMS-CPR is applied. Ideal: linewidth of both Tx and LO lasers are $0 \mathrm{~Hz}$. (a) Transmitter: $170 \mathrm{MHz}$, local oscillator: $0 \mathrm{~Hz}$; (b) Transmitter = local oscillator: $5 \mathrm{MHz}$.

\section{Conclusions}

In this work, an orthogonally polarized RF pilot tone is investigated to suppress the LPN and EEPN in long-haul coherent optical fiber communication systems. A 28-Gsym/s QPSK optical transmission system is numerically implemented, where the CPR is performed using the one-tap NLMS and DPD methods, respectively. Our results demonstrate that the LPN in the communication system can be completely eliminated and the EEPN can also be effectively suppressed, when the RF pilot tone scheme is applied prior to the CPR.

Author Contributions: Theoretical analyses and numerical simulations were implemented by T.X. The paper was mainly written by T.X., C.J. wrote some parts and edited the manuscript. S.Z. and M.L. made modifications. All authors reviewed and discussed the paper.

Funding: This work is in parts supported by the EU H2020 RISE Grant DAWN4IoE (778305) and the UK EPSRC Program Grant TRANSNET (EP/R035342/1).

Conflicts of Interest: The authors declare no conflict of interest.

\section{References}

1. Kaminow, I.; Li, T.; Willner, A.E. Optical Fiber Telecommunications VIA: Components and Subsystems, 6th ed.; Academic Press Publisher: Oxford, UK, 2013.

2. Ip, E.; Lau, A.P.T.; Barros, D.J.F.; Kahn, J.M. Coherent detection in optical fiber systems. Opt. Express 2008, 16, 753-791. [CrossRef] [PubMed]

3. Winzer, P.J.; Essiambre, R.J. Advanced modulation formats for high-capacity optical transport networks. J. Lightwave Technol. 2006, 24, 4711-4728. [CrossRef]

4. Savory, S.J. Digital coherent optical receivers: Algorithms and subsystems. IEEE J. Sel. Top. Quantum Electron. 2010, 16, 1164-1179. [CrossRef]

5. Zhao, J.; Liu, Y.; Xu, T. Advanced DSP for coherent optical fiber communication. App. Sci. 2019, 9, 4192. [CrossRef]

6. Mori, Y.; Zhang, C.; Igarashi, K.; Katoh, K.; Kikuchi, K. Unrepeated 200 km transmission of 40-Gbit/s 16-QAM signals using digital coherent receiver. Opt. Express 2009, 17, 1435-1441. [CrossRef]

7. Nakamura, M.; Kamio, Y.; Miyazaki, T. Pilot-carrier based linewidth-tolerant 8PSK self-homodyne using only one modulator. In Proceedings of the 33rd European Conference and Exhibition of Optical Communication, Berlin, Germany, 16-20 September 2007.

8. Cai, Y.; Gao, X.; Ling, Y.; Xu, B.; Qiu, K. RF pilot tone phase noise cancellation based on DD-MZM SSB modulation for optical heterodyne RoF link. Opt. Commun. 2020, 454, 124502. [CrossRef]

9. Jacobsen, G.; Xu, T.; Popov, S.; Li, J.; Friberg, A.T.; Zhang, Y. Receiver implemented RF pilot tone phase noise mitigation in coherent optical nPSK and nQAM systems. Opt. Express 2011, 19, 14487-14494. [CrossRef] 
10. Shieh, W.; Ho, K.P. Equalization-enhanced phase noise for coherent detection systems using electronic digital signal processing. Opt. Express 2008, 16, 15718-15727. [CrossRef]

11. Xie, C. Local oscillator phase noise induced penalties in optical coherent detection systems using electronic chromatic dispersion compensation. In Proceedings of the 2009 Conference on Optical Fiber Communication-Incudes post deadline papers, San Diego, CA, USA, 29 May 2009.

12. Lau, A.P.T.; Shen, T.S.R.; Shieh, W.; Ho, K.P. Equalization-enhanced phase noise for $100 \mathrm{~Gb} / \mathrm{s}$ transmission and beyond with coherent detection. Opt. Express 2010, 18, 17239-17251. [CrossRef]

13. Xu, T.; Liga, G.; Lavery, D.; Thomsen, B.C.; Savory, S.J.; Killey, R.I.; Bayvel, P. Equalization enhanced phase noise in Nyquist-spaced superchannel transmission systems using multi-channel digital back-propagation. Sci. Rep. 2015, 5. [CrossRef]

14. Zhuge, Q.; Xu, X.; El-Sahn, Z.A.; Mousa-Pasandi, M.E.; Morsy-Osman, M.; Chagnon, M.; Qiu, M.; Plant, D.V. Experimental investigation of the equalization-enhanced phase noise in long haul 56 Gbaud DP-QPSK systems. Opt. Express 2012, 20, 13841-13846. [CrossRef] [PubMed]

15. Fatadin, I.; Savory, S.J. Impact of phase to amplitude noise conversion in coherent optical systems with digital dispersion compensation. Opt. Express 2010, 18, 16273-16278. [CrossRef] [PubMed]

16. Kakkar, A.; Navarro, J.R.; Schatz, R.; Louchet, H.; Pang, X.; Ozolins, O.; Jacobsen, G.; Popov, S. Comprehensive study of equalization-enhanced phase noise in coherent optical systems. J. Lightwave Technol. 2015, 33, 4834-4841. [CrossRef]

17. Xu, T.; Jacobsen, G.; Popov, S.; Li, J.; Friberg, A.T.; Zhang, Y. Carrier phase estimation methods in coherent optical transmission systems influenced by equalization enhanced phase noise. Opt. Commun. 2013, 293, 54-60. [CrossRef]

18. Colavolpe, G.; Foggi, T.; Forestieri, E.; Secondini, M. Impact of phase noise and compensation techniques in coherent optical systems. J. Lightwave Technol. 2011, 29, 2790-2800. [CrossRef]

19. Xu, T.; Jacobsen, G.; Popov, S.; Li, J.; Friberg, A.T.; Zhang, Y. Analytical estimation of phase noise influence in coherent transmission system with digital dispersion equalization. Opt. Express 2011, 19, 7756-7768. [CrossRef]

20. Xu, T.; Jacobsen, G.; Popov, S.; Li, J.; Wang, K.; Friberg, A.T. Normalized LMS digital filter for chromatic dispersion equalization in 112-Gbit/s PDM-QPSK coherent optical transmission system. Opt. Commun. 2010, 283, 963-967. [CrossRef]

21. Fatadin, I.; Ives, D.; Savory, S.J. Differential carrier phase recovery for QPSK optical coherent systems with integrated tunable lasers. Opt. Express 2013, 21, 10166-10171. [CrossRef]

22. Savory, S.J. Digital filters for coherent optical receivers. Opt. Express 2008, 16, 804-817. [CrossRef]

23. Kudo, R.; Kobayashi, T.; Ishihara, K.; Takatori, Y.; Sano, A.; Miyamoto, Y. Coherent optical single carrier transmission using overlap frequency domain equalization for long-haul optical systems. J. Lightwave Technol. 2009, 27, 3721-3728. [CrossRef]

24. Jacobsen, G.; Xu, T.; Popov, S.; Sergeyev, S. Study of EEPN mitigation using modified RF pilot and Viterbi-Viterbi based phase noise compensation. Opt. Express 2013, 21, 12351-12362. [CrossRef] [PubMed]

25. Ly-Gagnon, D.S.; Tsukamoto, S.; Katoh, K.; Kikuchi, K. Coherent detection of optical quadrature phase-shift keying signals with carrier phase estimation. J. Lightwave Technol. 2006, 24, 12-21. [CrossRef]

26. Viterbi, A.J.; Viterbi, A.M. Nonlinear estimation of PSK-modulated carrier phase with application to burst digital transmission. IEEE Trans. Inf. Theory 1983, 29, 543-551. [CrossRef]

27. Liga, G.; Xu, T.; Alvarado, A.; Killey, R.I.; Bayvel, P. On the performance of multichannel digital backpropagation in high-capacity long-haul optical transmission. Opt. Express 2014, 22, 30053-30062. [CrossRef] [PubMed]

28. Maher, R.; Xu, T.; Galdion, L.; Sato, M. Spectrally shaped DP-16QAM super-channel transmission with multi-channel digital back propagation. Sci. Rep. 2015, 5, 08214. [CrossRef] [PubMed]

29. Ho, K.P.; Lau, A.P.T.; Shieh, W. Equalization-enhanced phase noise induced timing jitter. Opt. Lett. 2011, 36, 585-587. [CrossRef]

30. Nakamura, M.; Kamio, Y.; Miyazaki, T. Linewidth-tolerant real-time 40-Gbit/s 16-QAM self-homodyne detection using a pilot carrier and ISI suppression based on electronic digital processing. Opt. Lett. 2010, 35, 13-15. [CrossRef]

31. Nakamura, M.; Kamio, Y.; Miyazaki, T. Linewidth-tolerant real-time 10-Gbit/s 16-QAM transmission using a pilot-carrier based phase-noise cancelling technique. Opt. Express 2008, 16, 10611-10616. [CrossRef] 
32. Randel, S.; Adhikari, S.; Jansen, S.L. Analysis of RF-pilot-based phase noise compensation for coherent optical OFDM systems. IEEE Photon. Technol. Lett. 2010, 22, 1288-1290. [CrossRef]

33. Ip, E.; Kahn, J.M. Digital equalization of chromatic dispersion and polarization mode dispersion. J. Lightwave Technol. 2007, 25, 2033-2043. [CrossRef]

34. Liga, G.; Czegledi, C.B.; Xu, T.; Agrell, E.; Killey, R.I.; Bayvel, P. Ultra-wideband nonlinearity compensation performance in the presence of PMD. In Proceedings of the 42nd European Conference on Optical Communication, Dusseldorf, Germany, 5 December 2016.

(C) 2019 by the authors. Licensee MDPI, Basel, Switzerland. This article is an open access article distributed under the terms and conditions of the Creative Commons Attribution (CC BY) license (http://creativecommons.org/licenses/by/4.0/). 